\title{
SOLUBILIZATION OF DPPC SMALL UNILAMELLAR LIPOSOMES BY SUCROSE ESTERS. A FLUORESCENCE STUDY
}

\author{
EDUARDO BERRIOS, ANTONIO L. ZANOCCO, ELSE LEMP, GERMÁN GÜNTHER* \\ Universidad de Chile, Facultad de Ciencias Químicas y Farmacéuticas, Departamento de Química Orgánica y Fisicoquímica, \\ Casilla 233, Santiago 1, Chile \\ (Received: April 15, 2008 -Accepted: July 30, 2008)
}

\begin{abstract}
The effect of the microenvironment on the Laurdan fluorescence was employed to study the solubilization mechanism of Dipalmitoyl-sn-glycero-3phosphocholine (DPPC) small unilamellar vesicles with several sucrose monoesters. The saturation and the solubilization concentrations $\left(\mathrm{C}_{\mathrm{sat}}\right.$ and $\left.\mathrm{C}_{\mathrm{sol}}\right)$, the ester-DPPC molar ratio $\left(\mathrm{R}_{\mathrm{e}}\right)$ and the bilayer/aqueous partition coefficients $(\mathrm{K})$ were measured by monitoring changes in laurdan generalized polarization for four different alkyl esters of sucrose. All critical concentrations showed linear dependence with DPPC liposomes concentration. The saturation and the solubilization capacity of this type of surfactant do not show a direct dependence with the length of its alkyl chain. For the studied series the lauryl sucrose derivative is the compound which presents the higher capacity to saturate and solubilize the DPPC vesicles, while the capacity of the esters with shorter and longer alkyl chains worsen. When the results obtained for DPPC liposomes are compared with previously obtained data for DODAC a different structuration of sucrose moieties in the bilayer can be postulated.
\end{abstract}

Keywords: Liposomes solubilization, sucrose esters, Laurdan, lauryl sucrose, palmityl sucrose, myristyl sucrose, stearyl sucrose, SUVs

\section{INTRODUCTION}

Great attention has been paid to understanding the principles governing the interaction of different surfactants with lipids, starting from simple model of membranes such as liposomes and vesicles ${ }^{1-5}$ to more complex systems like stratum corneum. ${ }^{6}$ Currently the physical model relates the ability of surfactants to saturate and solubilize the bilayer, with its critical micellar concentration, $\mathrm{cmc}$, and its distribution coefficients, $\mathrm{K}$.

The most employed compounds to solubilize lipidic membranes correspond to the alkyl glucoside family, specially the octyl glucoside, ${ }^{1,8-13}$ a non-ionic surfactant with a relatively high critical micellar concentration, 18$25.3 \mathrm{mM} .^{6,14}$ Alkyl glucosides with different lengths of alkyl chain have also been employed as surfactants. ${ }^{3,6}$ Replacement of the octyl chain by longer alkyl groups improves the stability to hydrolysis and diminishes the toxicity of these kind of compounds. ${ }^{15}$ The surface-activity of alkyl glucosides is similar to that described for commercial mixtures of sugar esters, a related family of non-toxic, skin compatible, non-polluting and biodegradable surfactants. We are interested in evaluating the solubilization properties of pure sucrose esters towards different types of bilayers, by employing fluorescence methods.

Theamphiphilicfluorophore6-dodecanoyl-2-(dimethylamino)-naphthalene (Laurdan), incorporates into membranes with the fluorescent moiety localized in the region of the acyl bonds on the phospholipidic glycerol backbone. ${ }^{16}$ The spectroscopic behavior of this probe in the microaggregate has been related to the membrane polarity and/or the membrane fluidity (gel or fluid lamellar phases), ${ }^{17}$ because of its sensitivity to the solvent dielectric relaxation effect. As a consequence any change in the microenvironment sensed by Laurdan is reflected in its emission spectra, ${ }^{18}$ allowing its use as a useful tool in observing physico-chemical micro-properties in compartmentalized systems. To account for the emission spectra shifting of Laurdan, a currently used parameter is the generalized polarization, GP. ${ }^{17,19,20}$ High values of GP are ascribed to a less fluid surroundings, where water penetration is blocked or limited..$^{20}$

In a previous study we reported the sublytic alterations and the solubilization process of DODAC unilamellar vesicle bilayers upon addition of sucrose esters of different alkyl chain lengths. For DODAC vesicles, the incorporation of sucrose ester as co-surfactant promotes structural changes in the liposome bilayer. The voluminous sucrose head group locates on the surface, acting as a physical barrier that blocks the access of water molecules to the bilayer, additionally water molecules are more structured at the interface than the pure DODAC vesicles. The effects of the incorporation of sucrose ester to DODAC vesicles lead to changes in the polarity and fluidity of the probe surroundings. Consequently, Laurdan spectrum (GP value) changes. For DPPC liposomes the zwitterionic phosphocholine heads would build a different interface, and as consequence a different behavior is expected. Sucrose esters are very prone to saturate the bilayer of DODAC vesicles, but a significant quantity of surfactant is needed to solubilize the vesicle. ${ }^{21,22}$

In the present study we report on the solubilization of DPPC small unilamellar vesicles by four sucrose esters with different alkyl chain lengths, (capryl $\mathrm{C}_{9} \mathrm{H}_{10} \mathrm{CO}$ - , lauryl $\mathrm{C}_{11} \mathrm{H}_{23} \mathrm{CO}$-, miristyl, $\mathrm{C}_{13} \mathrm{H}_{27} \mathrm{CO}-$, and palmityl, $\mathrm{C}_{15} \mathrm{H}_{31} \mathrm{CO}-$ ). The effective molar ratio of surfactant to DPPC in the bilayers
(R) and the partitioning coefficient $(\mathrm{K})$ at different critical stages of the solubilization process were determined. The results obtained provide useful information about the effect the bulky hydrophilic head, the sucrose moiety and on the influence of the effect of the lipophillic hydrophilic balance on the capacity of these compounds to saturate and solubilize DDPC vesicles.

\section{EXPERIMENTAL}

Chemicals. The sucrose monoesters, $\beta$-D-fructofuranosyl-6-O-capryl- $\alpha$-Dglucopyranoside (MCS) $\beta$-D-fructofuranosyl-6-O-lauryl- $\alpha$-D-glucopyranoside (MLS), $\quad \beta$-D-fructofuranosyl-6-O-miristyl- $\alpha$-D-glucopyranoside (MMS), and $\beta$-D-fructofuranosyl-6-O-palmityl- $\alpha$-D-glucopyranoside (MPS) were synthesized by a modification of the Osipow-Snell method $^{23}, 24$ that yields a relatively complex mixture of mono- (mainly $6-\mathrm{O}$ and presumably $1-\mathrm{O}$ ), diand tri-esters. To isolate monoesters, chromatography on silica column was employed. Briefly, the reaction mixture solubilized in chloroform was eluted from a semi-preparative silica gel column by using chloroform:methanol: water (20:5:0.7). Thin layer chromatography (using the same mobile phase and staining with a butanolic solution of urea-ortophosphoric acid) showed only one compound in the purified sample. The NMR spectra, obtained on a Bruker ADX 300 spectrometer, with $\mathrm{DMSO}_{\mathrm{d}-6}$ containing $5 \%$ of $\mathrm{CH}_{3} \mathrm{OD}$ to avoid micellization, are in good agreement with previously reported spectra of monoesters. ${ }^{25}$ 1,2-Dipalmitoyl-sn-glycero-3-phosphocholine (DPPC) from Sigma and Laurdan from Molecular Probes were used as received. Solvents from Merck were HPLC quality. Water was treated with Milli-Q equipment from Waters.

Liposome preparation. Small unilamellar liposomes (SUVs) were obtained by ultrasonication of a 10mM DPPC solution in Milli-Q water, with a Cole Parmer Ultrasonic Homogenizer. Final concentrations were fixed by water addition. DPPC SUV's with different sucrose ester concentrations were obtained by adding small aliquots of concentrated sucrose ester solutions in water. Mixtures did not show spectral differences after been allowed to stand for 24 hours to equilibrate, as it has been previously reported. ${ }^{3}$

Fluorescence spectroscopy. Steady state fluorescence measurements of Laurdan were accomplished in a Fluorolog Tau-2 spectrofluorometer (SPEX, Jobin Ybon) at $25.0 \pm 0.5^{\circ} \mathrm{C}$. The uncorrected values of generalized polarization of Laurdan $\left(3 \mu \mathrm{M}, \lambda_{\mathrm{ex}}=364 \mathrm{~nm}, \lambda_{\mathrm{em}}=440 \mathrm{~nm}\right.$ and $\left.490 \mathrm{~nm}\right)$, were determined by using the expression of Parassasi et al., Eq (1): ${ }^{18,20}$

$$
G P=\frac{\left(\left.\right|_{440}-\left.\right|_{490}\right)}{\left(f_{440}+\left.\right|_{490}\right)}
$$

Determination of the effective molar ratio of surfactant to lipid in the bilayers $\left(\mathrm{R}_{\mathrm{e}}\right.$ ) and the partitioning coefficient $(\mathrm{K})$. The equilibrium partition model $1^{7}, 26,27$ predicts a partitioning coefficient $(\mathrm{K})$ for the distribution of a surfactant between the lipid bilayer and the aqueous media. In a vesicular 
solution this parameter is defined as:

$$
K=\frac{C_{\theta}}{\left[\left(L+C_{\theta}\right) C_{W W^{\prime}}\right]}\left[\mathrm{mM}^{-1}\right]
$$

where $\mathrm{C}_{\mathrm{B}}$ and $\mathrm{C}_{\mathrm{W}}$ are the $\mathrm{mM}$ surfactant concentrations in the lipidic and aqueous pseudo phases, respectively and $\mathrm{L}$ is the lipid concentration (DPPC in our experiments). When the surfactant concentration in the bilayer is in the same order of the lipid concentration Eq. (2) can be rewritten as:

$$
K=\frac{\mathrm{Re}}{\left[(1+\mathrm{Re}) \mathrm{C}_{\mathrm{w}}\right]^{\left[\mathrm{mM}^{-1}\right]}}
$$

where Re corresponds to the effective molar ratio of surfactant to lipid in the bilayers $\left(\mathrm{C}_{\mathrm{B}} / \mathrm{L}\right)$.

Solubilization of liposomes or vesicles can be characterized by two critical parameters, $C^{\text {sat }}$ and $\mathrm{C}^{\text {sol }} . \mathrm{C}^{\text {sat }}$ is the surfactant concentration necessary to saturate the liposome bilayer, and $\mathrm{C}^{\text {sol }}$ is the concentration needed to complete the bilayer solubilization into mixed micelles. In static light scattering measurements for liposome solubilization, these parameters correspond to breakpoints observed when data are plotted against surfactant concentration. According to previous works, ${ }^{1,3,27,28}$ both critical concentrations exhibit a linear dependence on the lipid or phospholipid concentration, as stated by Eq. (4). This relation allows the determination of the effective surfactant to lipid molar ratios for surfactantsaturated vesicles $\left(\mathrm{R}_{\mathrm{e}}^{\text {sat }}\right)$ and for lipid saturated mixed-micelles $\left(\mathrm{R}_{\mathrm{e}}^{\text {sol }}\right)$ as well as the concentration of monomeric surfactant $\left(\mathrm{C}_{\mathrm{w}}\right)$ coexisting with the aggregates.

$$
\left.C_{t}^{c}=C_{m}^{c}+R_{e}^{c} L \mid m M^{-1}\right\rfloor
$$

In Eq. 4, $\mathrm{C}_{\text {t }}$ is the total surfactant concentration at each breakpoint. The superindexes indicate the different critical points, saturation and solubilization.

\section{RESULTS AND DISCUSSION}

The solubilization of DPPC vesicles by the addition of four different sucrose esters was monitored by using GP. For all the surfactants employed (capryl, lauryl, miristyl and palmityl sucrose esters) the shape of the solubilization profiles (plotting obtained GP values) is fully comparable: (1) An initial stage where the GP value remains constant, independent of surfactant concentration, to reach a breakpoint. This point is related with the saturation of liposome bilayer, and the concentration of surfactant at this point corresponds to $\mathrm{C}_{\mathrm{t}}^{\text {sat }}$. (2) After the breakpoint, further addition of surfactant leads to a decrease in GP value, at these sucrose ester concentrations mixed micelles and saturated liposomes coexist. The GP decrease follows until a new breakpoint is reached. At this point, solubilization of vesicles is complete and the surfactant concentration is denoted as $\mathrm{C}_{\mathrm{t}}^{\text {sol }}$. Beyond this point no changes in GP were observed upon further sucrose ester addition. Figure 1 shows the change in the GP value of laurdan labeled DPPC SUVs, when allowed to equilibrate with different concentrations of MCS.

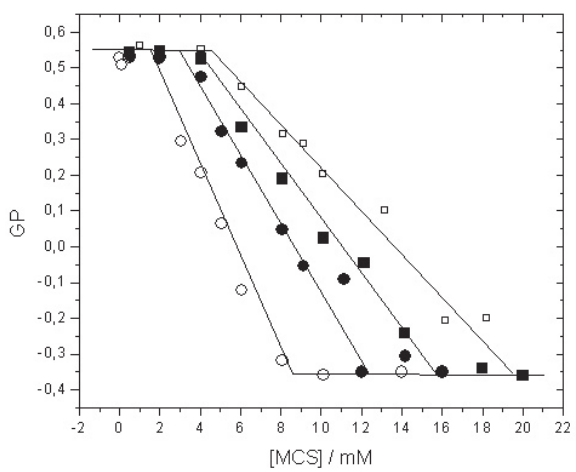

Fig. 1 Solubilization profile of DPPC liposomes $0.2(\circ), 0.4(\bullet), 0.6($ ) and $1.0 \mathrm{mM}(\square)$ with MCS at $20^{\circ} \mathrm{C}$.
Plots of Laurdan GP against surfactant concentration at different DPPC liposome concentrations (between 0.1 to $1.0 \mathrm{mM}$ ) allow the graphical determination of the critical surfactant concentrations $\mathrm{C}_{\mathrm{t}}^{\text {sat }}$ and $\mathrm{C}_{\mathrm{t}}^{\text {sol }}$ at each DPPC liposomes concentration. This methodology, where the fluorescent probe response is plotted against the concentration of surfactant, is analogous to the one employed when the measured property is scattered light. ${ }^{6}$

In order to determine the surfactant to lipid molar ratio, $\mathrm{R}_{\mathrm{e}}$, and the free surfactant concentration, $\mathrm{C}_{w}$, the previously determined critical surfactant concentrations were plotted against DPPC concentration (Eq. 4). The data show a good linear fit, (as can be seen in Figure 2) allowing to obtain R values from the slope. $\mathrm{C}_{w}$ values can be determined from the intercept, but they have a large error, in some cases being far from the corresponding $\mathrm{cmc}$ value. The reason for the poor agreement between $\mathrm{C}_{\mathrm{w}}$ and $\mathrm{cmc}$ is probably due to the low cmc values for this family of non-ionic surfactants (Table 2). The experimental constrain, the surfactant concentration must be on the order of lipid concentration, forces surfactant concentrations to be higher than its own cmc. Thus, this 'far' extrapolation, may be the origin of the uncertainty observed in the values for the intercept.

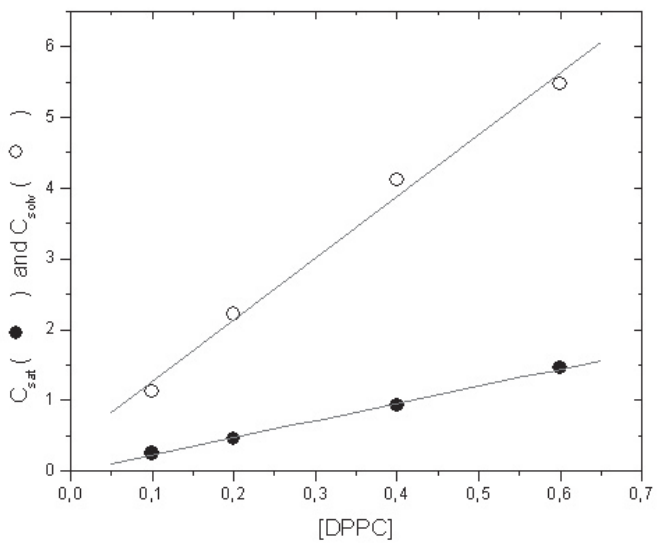

Fig. 2 Plot of solubilization and saturation critical concentrations $\left(\mathrm{C}_{f}^{\text {sol }}(\mathrm{O})\right.$ and $\left.\mathrm{C}_{\mathrm{t}}^{\text {sat }}(\bullet)\right)$ against DPPC concentration when MPS is employed as surfactant at $20^{\circ} \mathrm{C}$.

Table 1 shows the values for $\mathrm{R}_{\mathrm{e}}$ determined for all the different studied surfactants at both critical points, bilayer saturation and vesicle solubilization. As was mentioned these values were obtained from the fitting of data by using Eq. 4. The lauryl derivative shows the largest solubilization capacity meanwhile the capryl derivative (with a shorter alkyl chain) shows the lowest. For the longer derivatives, the expected decrease of saturation and solubilization capacity upon increase of the number of methylenic units is observed. Previously reported data for the solubilization of phosphatidylcholine liposomes and liposomes formed with lipids of stratum corneum employing alkylglucosides, ${ }^{3,}$ ${ }^{6}$ show a similar behavior. For the solubilization of stratum corneum the $\mathrm{R}$ values show a minimun for n-nonyl- $\beta$-D-glucopyranoside, and the shorter n-octyl derivative shows a slightly higher value. In Figure 3 the values for $\mathrm{R}^{\text {sol }}$ of three different systems are plotted: Alkyl glucoside: DPPC liposomes, ${ }^{3}$ Alkyl glucosides: Stratum Corneum lipid liposomes, ${ }^{6}$ and sucrose ester: DPPC liposomes. From this plot several observations can be made: (1) Sucrose esters with more than eleven methylenic units are more adequate solubilizing agents than the corresponding alkyl glucosides. Less quantity of surfactant is required to solubilize one mol of liposomes. (2) Two of the included systems show a minimum for $\mathrm{R}_{\mathrm{e}}$, indicating the existence of a balance between two opposite factors, the detergent $\mathrm{cmc}$ and to the affinity of the detergent with the particular system of liposomes. The overall balance is responsible of the ability of the specific surfactant to solubilize (saturate) the liposomes.

Table 1 Surfactant to DPPC molar ratios $(R e)$ for the bilayer saturation and solubilization of sucrose esters with DPPC small unilamellar vesicles.

\begin{tabular}{|c|c|c|}
\hline & $\mathrm{R}_{\mathrm{e}}^{\text {sat }}$ & $\mathrm{R}_{\mathrm{e}}^{\text {sol }}$ \\
\hline MCS & $3.90 \pm 0.30$ & $9.20 \pm 0.62$ \\
\hline MLS & $1.10 \pm 0.02$ & $3.40 \pm 0.09$ \\
\hline MMS & $1.70 \pm 0.08$ & $4.40 \pm 0.32$ \\
\hline MPS & $2.40 \pm 0.10$ & $8.60 \pm 0.13$ \\
\hline
\end{tabular}


As a result, the surfactant structure is critical, above certain alkyl chain length the presence of a sucrose moiety instead a glucose enhances the activity against liposomes, but below this limit the balance between the hydrophilic and lipophilic counterparts make the detergent a very inadequate candidate to be used in solubilization procedures.

Evaluation of the bilayer/aqueous phase partitioning coefficients, $\mathrm{K}$, with Eq. 3, requires knowing the free surfactant concentration, $\mathrm{C}_{\mathrm{w}}$. The uncertainty in the experimental determined values for this parameter could be overcome by using cmc instead of $\mathrm{C}_{\mathrm{w}}$ values. This approach is supported by previous data showing that free surfactant concentrations are always close to those of cmc. ${ }^{3}$ ${ }^{6}$ The solubilization process of liposomes is mainly ruled by the formation of mixed micelles, and in addition the values for free surfactant concentrations are no more than $3 \%$, which is below the cmc value for saturation and over the $\mathrm{cmc}$ value for solubilization.

The bilayer/aqueous phase partitioning coefficients calculated with these approaches and the cmc values ${ }^{29}$ are shown in Table 2 . These data indicate an increasing affinity of the sucrose ester to the vesicle bilayer with the increase of the length of the alkyl chain, but besides this, the quantity of surfactant needed to saturate and solubilize the vesicle increases significantly. Comparison of the ratio between $\mathrm{K}^{\text {sat }}$ and $\mathrm{K}^{\text {sol }}$ for each surfactant shows again a non direct dependence with the surfactant alkyl chain length, indicating that both the partitioning of surfactants into vesicles and the complete solubilization depend not only on the alkyl chain length. The partitioning is still mainly ruled by the surfactant cmc values, as has previously been reported for alkylglucosides, ie. by the hydrophilic-lipophilic balance in the surfactant molecule.

Table 2 Partition coefficients (K), in $\mathrm{mM}$ units, for the bilayer saturation and solubilization of sucrose esters with DPPC small unilamellar vesicles. Cmc values for all sucrose esters at $25^{\circ} \mathrm{C}$, previously reported, are included.

\begin{tabular}{|c|c|c|c|}
\hline & $\mathrm{K}^{\text {sat }} / \mathrm{mM}^{-1}$ & $\mathrm{~K}^{\text {sol }} / \mathrm{mM}^{-1}$ & $\mathrm{cmc} / \mathrm{mM}^{29}$ \\
\hline MCS & 0.44 & 0.50 & 1.80 \\
\hline MLS & 1.50 & 2.2 & 0.35 \\
\hline MMS & 26.00 & 34.0 & 0.024 \\
\hline MPS & 88.00 & 112.0 & 0.008 \\
\hline
\end{tabular}

An additional relevant aspect to be considered corresponds to the applicability of sucrose esters in solubilization of biological membranes. Comparison of the MLS concentration needed to saturate and solubilize DPPC SUV's with data for docecyl glucoside-phosphatidylcholine liposomes (Figure 3) shows that when using MLS and DPPC SUV's a concentration of approximately near to one half than that needed of dodecyl glucoside produces bilayer saturation and the same proportion is necessary to reach the saturation critical point. These findings show MLS as a very adequate surfactant to be used in membrane solubilization processes. Consequence of hydrophiliclipophilic balance the shorter sucrose ester, MCS has worse properties than the homologous decyl glucoside, as previously mentioned.

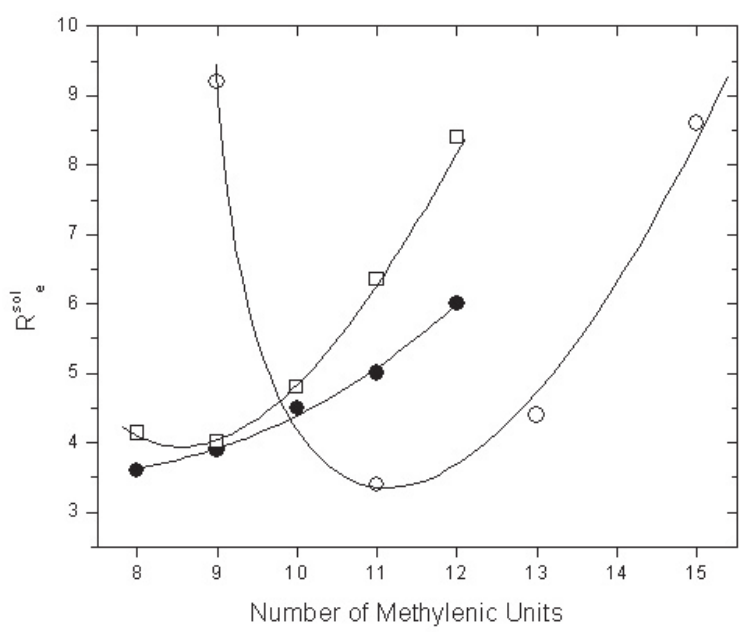

Fig. 3 Plot of $\mathrm{R}_{\mathrm{e}}^{\text {sol }}$ for three different systems: Alkyl glucoside: DPPC liposomes (•), ${ }^{3}$ Alkyl glucosides: Stratum Corneum lipid liposomes $(\square),{ }^{6}$ and sucrose ester: DPPC liposomes (o).
And additional point of interest arises from the comparison of solubilization profiles of DODAC ${ }^{22}$ and DPPC vesicles with sucrose esters. Both systems show a different interface, small cationic heads for DODAC and big zwitterionic heads for DPPC. Figure 4 shows the solubilization profile when the palmityl derivative (MPS) is used. The differences are readily detectable, the incorporation of sucrose ester on DPPC liposomes at sublytic concentrations does not originate noticeable changes in the surroundings of the probe, and the value of GP remains constant. At work temperature, DPPC liposomes are tight, so the presence of sucrose esters does not enhance the water blocking. On the other hand the presence of ester in the case of DODAC vesicles promotes an important increase in the GP values (at least 0.5 units starting from -0.2 for neat vesicles). This important increase in GP has been ascribed to a significant blocking effect of sucrose moieties over vesicle surface. ${ }^{22}$

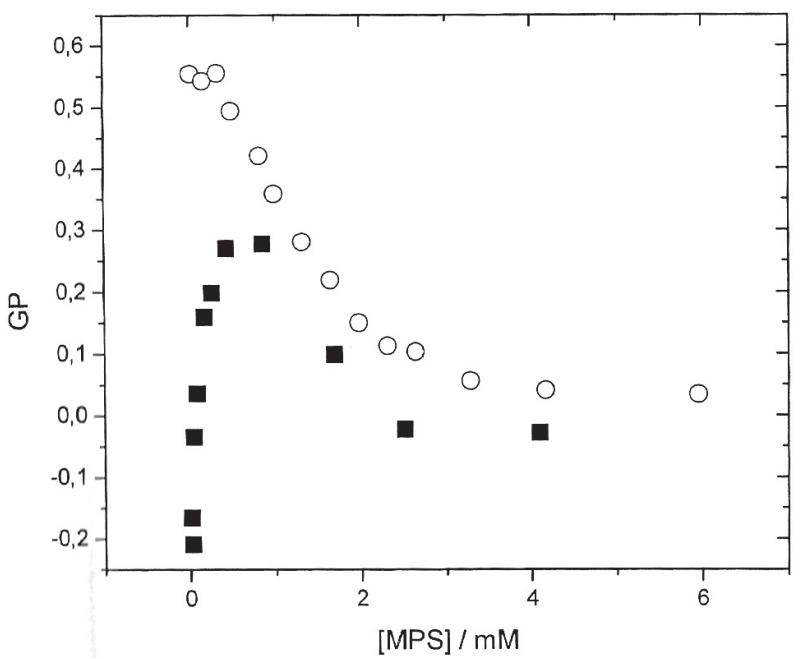

Figure 4 Solubilization profile of $0.2 \mathrm{mM}$ DPPC liposomes (o) and 0.2 $\mathrm{mM}$ DODAC vesicles (ש) with MPS at $20^{\circ} \mathrm{C}$.

\section{CONCLUSIONS}

Measurements of Laurdan generalized polarization enable fluorescence techniques to be used as an attractive, simple and useful option to determine the parameters associated with liposome solubilization by surfactants.

For the interaction of sucrose esters of different alkyl chain with DPPC SUVs, the $\mathrm{R}$ values do not depend directly on alkyl chain length, there is a specific size where the balance of the hydrophobic chain and hydrophilic head enhances the saturating and solubilizing capacity. Thus, for this surfactant series, the lauryl derivative is the most effective solubilizing agent and those with shorter and larger alkyl chains have worse capacities.

\section{ACKNOWLEDGEMENTS}

This work was supported by funds from Fondecyt 1040573.

\section{REFERENCES}

1. M. T. Paternostre, M. Roux, J. L. Rigaud, Biochemistry 27, 2668, (1988)

2. N. Deo, P. Somasundaran, Colloid Surface, A 186, 33, (2001)

3. O. Lopez, M. Cocera, J. L. Parra, A. De La Maza, Colloid Surface A, 193 , 221, (2001).

4. M. Ohnishi, H. Sagitani, J. Am. Oil Chem. Soc. 70, 679, (1993)

5. F. Nomura, M. Nagata, T. Inaba, H. Hiramatsu, H. Hotani, K. Takiguchi, P. Natl. Acad. Sci. USA 98, 2340, (2001)

6. O. Lopez, M. Cocera, J. L. Parra, A. De La Maza, Colloid Polym. Sci. 279, 909, (2001).

7. D. Lichtenberg, BBA 821, 470, (1985)

8. M. L. Jackson, C. F. Schmidt, D. Lichtenberg, B. J. Litman, A. D. Albert, Biochemistry 21, 4576, (1982)

9. O. Lopez, M. Cocera, P. Walther, A. De La Maza, L. Coderch, J. L. Parra, Colloid Surface A, 168, (2000)

10. O. Lopez, M. Cocera, L. Coderch, J. L. Parra, L. Barsukov, A. De La Maza, J. Phys. Chem. B 105, (2001) 
11. O. Lopez, M. Cocera, P. Walther, E. Wehrli, L. Coderch, J. L. Parra, A. De La Maza, Micron 32, 201, (2001)

12. M. Ueno, N. Hirota, H. Kashiwagi, S. Sagasaki, Colloid Polym. Sci. 282, (2003)

13. S. Morandat, K. El Kirat, Colloid Surface B-Biointerfaces 55, 179, (2007)

14. M. Frindi, B. Michels, R. Zana, J. Phys. Chem. 96, 8137, (1992)

15. M. T. Garcia, I. Ribosa, E. Campos, J. S. Leal, Chemosphere, 35, 545, (1997)

16. J. C.-M. Lee, R. J. Law, D. E. Discher, Langmuir 17, 3592, (2001)

17. M. Viard, J. Gallay, M. Vincent, M. Paternostre, Biophys. J. 80, 347, (2001)

18. T. Parasassi, G. De Stasio, G. Ravagnan, R. M. Rusch, E. Gratton, Biophys. J. 60, 179, (1991)

19. F. M. Harris, K. B. Best, J. D. Bell, BBA 1565, 123, (2002)

20. T. Parassasi, E. K. Krasnowska, L. Bagatolli, E. Gratton, J. Fluoresc. 8, $365,(1998)$.
21. E. Lemp, A. L. Zanocco, G. Gunther, Colloid Surface A, 229, 63, (2003)

22. N. Becerra, L. R. De La Nuez, A. L. Zanocco, E. Lemp, G. Gunther, Colloid Surface A 272, 2, (2006)

23. L. Osipow, F. D. Snell, W. C. York, A. Finchler, Ind. Eng. Chem. 48, 1459, (1956)

24. W. York, C. A. Finchler, L. Osipow, F. D. Snell, J. Am. Oil Chem. Soc. 33 424, (1956)

25. I. R. Vlahov, P. I. Vlahova, R. J. Lindhart, J. Carbohyd. Chem. 16, 1, (1997)

26. P. Schurtenberger, N. Mazer, W. Kanzig, J. Phys. Chem. 89, 1042, (1985)

27. S. Almog, B. J. Litman, W. Wimley, J. Cohen, E. J. Wachtel, Y. Barenholz, A. Benshaul, D. Lichtenberg, Biochemistry 29, 4582, (1990)

28. A. De La Maza, J. L. Parra, Langmuir 9, 870, (1993)

29. N. Becerra, C. Toro, A. L. Zanocco, E. Lemp, G. Günther, Colloid Surface, A $327,134,(2008)$ 